\title{
Tuning effect in particle masses and nuclear data
}

\section{Sergey Sukhoruchkin}

B. P. Konstantinov Petersburg Nuclear Physics Institute

E-mail: Sukhoruchkin_SI@pnpi.nrcki.ru

Recent analysis of nuclear binding energies and excitations of many magic nuclei allowed a confirmation of the tuning effect in particle masses. Stable mass/energy intervals coinciding or rationally connected with charge mass splitting of the nucleon $\delta m_{N}=1293.3 \mathrm{keV}$ and the lepton $m_{e}=511 \mathrm{keV}$ appear in the shift of the neutron mass relative to integer number of $m_{e}$. Using evaluated by CODATA exact ratio $1838.6836605(11)$ between masses of the neutron and the electron, we determine the shift $\delta m_{n}=161.65(6) \mathrm{keV}$ from integer number of $m_{e}$, namely $115 \delta-m_{e}$. Period $\delta=16 m_{e}$, determined here with very high precision, is the common for many particle masses, for example, $\mathrm{n}=13$ for the muon mass, $\mathrm{n}=16$ for $f_{\pi}, \mathrm{n}=17$ for the pion mass, $\mathrm{n}=18$ for a half of nucleon $\Delta$-excitation and $\mathrm{n}=115$ for the neutron mass. This shift in the neutron mass is in a ratio $8 \times 1.0001(1)$ with the nucleon mass difference $\delta m_{N}$. CODATA relation means that nucleon masses are: $m_{n}=115 \cdot 16 m_{e}-m_{e}-\delta m_{N} / 8$ and $m_{p}=115 \cdot 16 m_{e}-m_{e}-9 \delta m_{N} / 8$.

The shift of $m_{e}$ is presumably connected with the baryon number $\left(m_{e} / 3\right.$ per constituent quark) estimated in NRCQM (Nonrelativistic Constituent Quark Model) as $M_{q}=m_{\Xi}-/ 3=441$ $\mathrm{MeV}=3\left(\Delta M_{\Delta}=147 \mathrm{MeV}\right)$, three-fold value of the pion-exchange interaction in NRCQM. Together with the meson constituent quark mass $M_{q}^{\prime \prime}=m_{\rho} / 2=409 \mathrm{MeV}$, they are in ratios with the vector boson masses equal to the lepton ratio $\mathrm{L}=m_{\mu} / M_{e}=13 \cdot 16-1=207=M_{Z} / M_{q}=M_{W} / M_{q}^{\prime \prime}$. Simultaneously, the ratio between masses of vector Z-boson and the muon $m_{\mu} / M_{Z}=115.9 \cdot 10^{-5}$ is very close to QED-radiative correction $\alpha / 2 \pi=115.9 \cdot 10^{-5}$. Such factor with the QED parameter $\alpha=1 / 137$ was found between the scalar boson mass $M_{H}=126 \mathrm{GeV}$, the parameter $\Delta M_{\Delta}=147$ $\mathrm{MeV}$ and $m_{e} / 3=170 \mathrm{keV}$. V. Belokurov and D. Shirkov suggested that the same QED correction can be applied to the electron mass itself. Cumulative effect in the constituent quark mass $M_{q}=3 \cdot 18 \delta=3 \Delta M_{\Delta}$ could result in the value close to $m_{e}$ and could be connected with the origin of the mass $m_{e}$ from the physical condensate and the estimate of the Plank Mass value $M_{P}=L \Delta M_{\Delta}(\alpha / 2 \pi)^{-6}$. We draw attention to the five empirical relations based entirely on the unexpectedly accurate CODATA relation with the real electron mass and the period $16 m_{e}$.

38th International Conference on High Energy Physics

3-10 August 2016

Chicago, USA 
Recent analysis of particle masses and new nuclear data [1-3] confirmed CODATA relation between the nucleon $\left(m_{n}, m_{p}\right)$ and the electron $\left(m_{e}\right)$ masses. It is resulted in the presentation:

$m_{n}=115 \cdot 16 m_{e}-m_{e}-\delta m_{N} / 8$ and $m_{p}=115 \cdot 16 m_{e}-m_{e}-9 \delta m_{N} / 8$

with shifts $\delta m_{N} / 8=161 \mathrm{keV}$ and $m_{e} / 3=170 \mathrm{keV}$ corresponding to the fine structure with the period $\delta^{\prime}=9.5 \mathrm{keV}$ and integer numbers $\mathrm{n}=17,18$ (of this period). Such fine structure (with exactly the same period and $n=13-18$ ) was found in nuclear excitations of many near-magic nuclei. In Table 1 [1-3] these values are presented (in the bottom part) as parameters of the expression $n \cdot 16 \mathrm{~m}_{\mathrm{e}}(\alpha / 2 \pi)^{X} \mathrm{M}$ with $\alpha / 2 \pi=115.9 \cdot 10^{-5}$ (close to $1 / 32 \cdot 27=115.7 \cdot 10^{-5}$ ) and different values $\mathrm{X}, \mathrm{M}$ and $\mathrm{n}=1,13-18$.

Following five empirical correlations could be mentioned [1-5].

1) Besides relations $M_{Z}=\mathrm{L} M_{q}$ and $M_{W}=\mathrm{L} M_{q}^{\prime \prime}$ the masses of scalars $\left(M_{H}\right.$ and $M_{H}^{\prime \prime}=(2 / 3) m_{t}=2 M^{L 3}=116$ $\mathrm{GeV}$ ) can be estimated as $16 \cdot 18 M_{q}$ and $16 \cdot 16 M_{q}$ (Tables 1-3).

2) Well-known QED parameter for a short distance, $\alpha_{Z}=1 / 129$ (an analog of 1/137) can be used for the interconnection of $m_{e}, m_{\pi}$ and $m_{\pi} /(2 / 3) m_{t}=M_{H}^{\prime \prime}=115 \mathrm{GeV}=2 M^{L 3}=2.58 \mathrm{GeV}$ (unconfirmed mass groupings found by ALEPH and L-3 collaborations at CERN). These relation between the top quark and electron masses could be helpful for the understanding of the origin of the color.

3) Origin of the dark matter could be connected with the observed shifts in strange octet baryons (two bottom lines in Table 2) and above discussed interconnection between $m_{e}$ and heavy fermions. 4) Evolution of nucleon mass (Fig.1) from the initial value $3 M_{q}$ (top) to $6 f_{\pi}+\Delta M_{\Delta}=2 M_{q}^{\prime \prime}+\Delta M_{\Delta}$ (bottom) means the distinguished character of NRCQM-parameters based on QCD gluon quarkdressing effect (Fig.2 in [4]). Progress of nuclear physics in a view of the presence of correlations between $m_{e}$ and heavy fermions $M_{q}$ and $M_{q}^{\prime \prime}$ (plus $f_{\pi}, m_{\pi}, \Delta M_{\Delta}$ ) which are parameters of nuclear models is in line with F. Wilczek expectation about a future great role of "nuclear chemistry" [6].

5) Confirmation with nuclear data analysis of intervals/periods $\Delta$ (observed in particle masses, boxed in Table 2, including tau-lepton mass) put forward a problem of parameters interpretation.

Table 1. Representation of parameters of tuning effects in particle masses ( 3 top sections) and nuclear data (bottom) with the expression $\mathrm{n} \cdot 16 \mathrm{~m}_{\mathrm{e}}(\alpha / 2 \pi)^{X} \mathrm{M}$ with different values of $\mathrm{X}$-power at QED factor $\alpha / 2 \pi$ $(\alpha=1 / 137)$ and integers $M$ and $n=1,13-18$. Boxed are five groups of values differing with $\alpha / 2 \pi=115.9 \cdot 10^{-5}$.

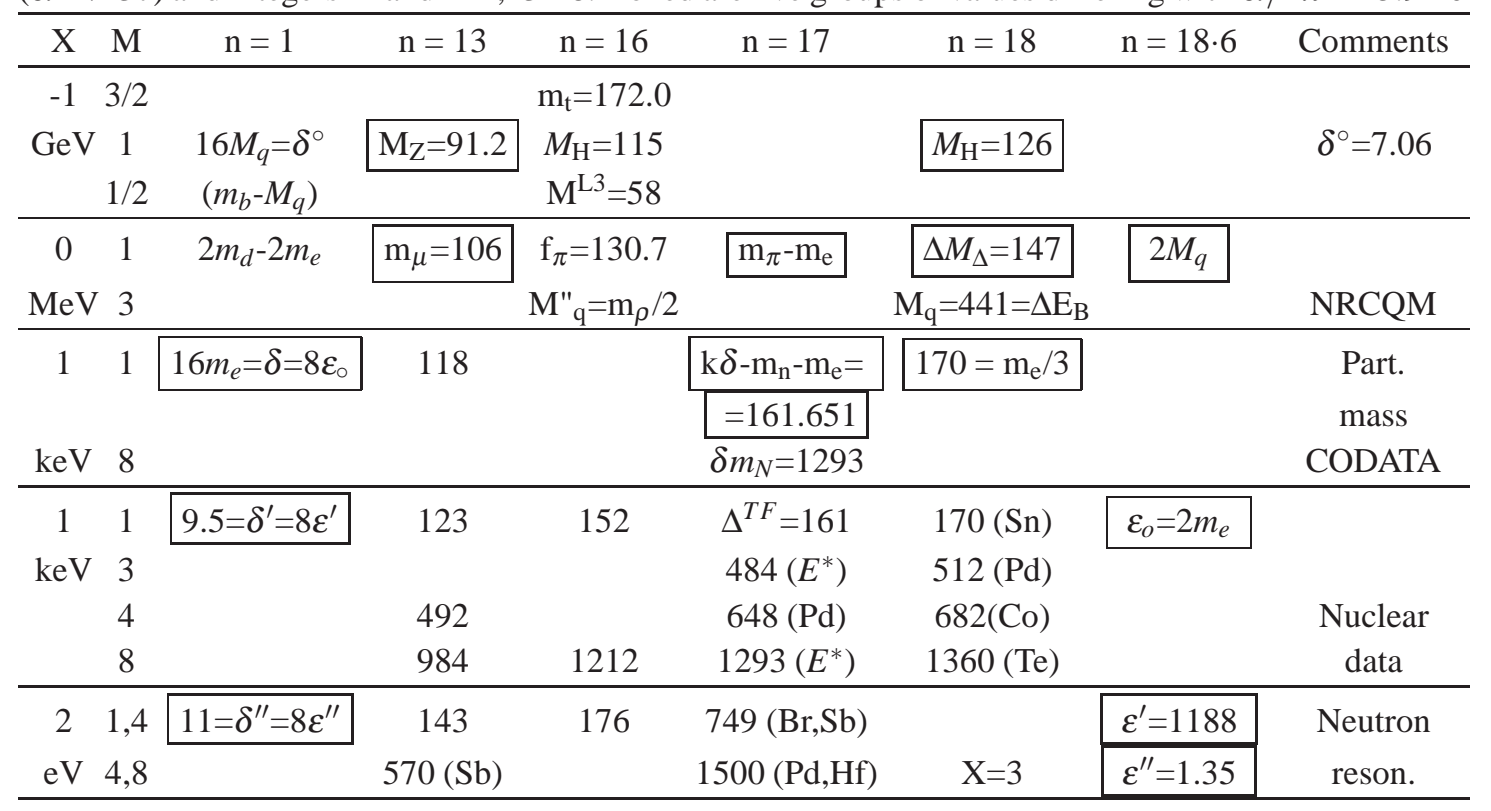




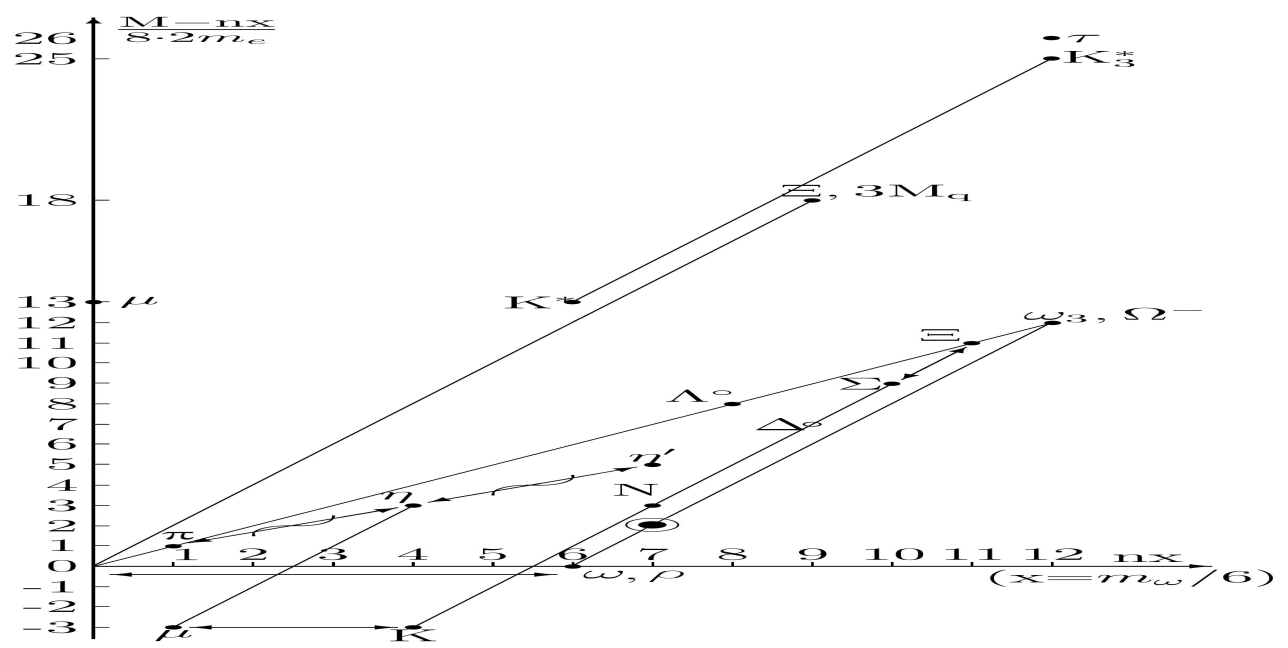

Fig. 1. Particle masses in two-dimensional representation [1-5]. Values along the horizontal axis are given in units $f_{\pi}=16 \cdot 16 m_{e}=130.8 \mathrm{MeV}=16 \delta$, remainders - on vertical axis in units $16 m_{e}=\delta$. Main lines correspond to $\Delta M_{\Delta}=147 \mathrm{MeV}=18 \delta$ - parameter of quark structure derived from nucleon $\Delta$ - excitation close to $(1 / 3)$ of initial mass of constituent quark $M_{q}$ and (1/9) of initial baryon mass $3 M_{q}=m_{\Xi}$ (top). Nucleon mass (N) is on the line from kaon mass ( $\mathrm{K}$ ) to hyperon mass $(\Xi)$. Nucleon mass within nucleus (circled point) is close to $6 f_{\pi}+\Delta M_{\Delta}$. Pion mass $140 \mathrm{MeV}=f_{\pi}+\delta$ is rational to masses of $\Lambda, \Xi, \Omega$ and is in equidistancy with pseudoscalars $m_{\eta^{\prime}}-m_{\eta}=m_{\eta}-m_{\pi}^{ \pm}$(crossed arrow). Tau lepton mass (top) is close to $12 f_{\pi}+2 m_{\mu}(\mathrm{n}=12 \cdot 16+2 \cdot 13)$.

Table 2. Comparison of particle masses with the period $16 m_{e}=\delta=8176 \mathrm{keV}$ (numbers of periods k).

\begin{tabular}{|c|c|c|c|c|c|}
\hline Particle & $\mathrm{m}_{\mathrm{i}}, \mathrm{MeV}$ & $\mathrm{k}$ & \multicolumn{2}{|c|}{$\mathrm{m}_{\mathrm{i}}-\mathrm{k} \cdot 16 \mathrm{~m}_{\mathrm{e}}$ Comments $($ in $\mathrm{MeV})$} & Comments \\
\hline$\mu$ & 105.65837 & 13 & -0.6294 & $-m_{e}-0.118$ & \\
\hline$\tau$ & $1776.8(2)$ & $4 \cdot 48 \cdot+2 \cdot 13$ & & $-5.6(2)$ & diff. $-2 m_{e}-(4.6 \mathrm{MeV}=\Delta)$ \\
\hline$f_{\pi}$ & $130.7(4)$ & 16 & & $\approx 0$ & \\
\hline$\pi^{ \pm}$ & $139.5702(4)$ & 17 & +0.57624 & $+m_{e}+0.065$ & \\
\hline$\Delta^{\circ}-\mathrm{n}$ & $294.2(2)$ & 36 & & $2\left(\Delta M_{\Delta}=147.1\right)$ & $\Delta E_{B}=147.3 \mathrm{MeV}$, Fig.4 in [4] \\
\hline$M_{q} \mathrm{NRCQM}$ & 441 & $3 \cdot 18$ & & & $\Delta E_{B}=441 \mathrm{MeV}$, Fig.4 in [4] \\
\hline$M_{H} / 18 \cdot 16$ & 436 & $3 \cdot 18-\Delta$ & & $-5=-\Delta$ & \\
\hline$M_{Z} / \mathrm{L}$ & 440.5 & $3 \cdot 18$ & & & diff. $\approx-2 m_{e}$ \\
\hline t-quark & $172000(1000)$ & $24 \times 16 \times 54$ & & & $169540 \mathrm{MeV}=24 \delta^{\circ}$ \\
\hline$\eta^{\prime}-\eta, \eta-\pi^{ \pm}$ & 409 & 50 & & $\approx 0$ & \\
\hline$M_{q}^{\Delta} \mathrm{NRCQM}$ & 410 & 50 & & & $\Delta E_{B}=409 \mathrm{MeV}$, Fig. 4 in [4] \\
\hline$\rho$ & $775.49(34)$ & 96 & $-9.40(34)$ & $-9.20=-2 \Delta$ & \\
\hline$M_{q}^{\prime \prime} \mathrm{NRCQM}$ & 387.7 & 48 & $m_{\rho} / 2$ & $-4.60=-\Delta$ & \\
\hline$M_{W} / \mathrm{L}$ & 388.4 & $3 \cdot 16$ & $3 f_{\pi}$ & & diff. $\approx-2 m_{e}$ \\
\hline $\mathrm{p}$ & $938.2720(1)$ & 115 & -1.96660 & & $-\mathrm{m}_{\mathrm{e}}-(9 / 8) \delta \mathrm{m}_{\mathrm{N}}$ \\
\hline $\mathrm{n}$ & $939.5654(1)$ & 115 & $-0.6726(1)$ & & $-\mathrm{m}_{\mathrm{e}}-(1 / 8) \delta \mathrm{m}_{\mathrm{N}}$ \\
\hline$\Sigma^{\circ}$ & $1192.64(2)$ & 146 & $-1.05(2)$ & $-0.51 \cdot 2=-1.02$ & \\
\hline$\Xi^{\circ}$ & $1314.86(20)$ & 161 & $-1.47(20)$ & $-0.51 \cdot 3=-1.53$ & \\
\hline
\end{tabular}

To explain the period of $\delta=16 m_{e}=2 \Delta-2 m_{e}$ (with $\Delta=9 m_{e}$ ) the symmetry motivated arguments were searched [1-5] starting with indications on the reality of integer ratios and long-range correlations in particle masses and nuclear data. Relation between the constituent quark mass $M_{q}=3 \Delta M_{\Delta}$ 
could be a reflection of the influence of the physical condensate [7] (and might be connected with the gravitation [8,9]). Analysis indicates on the existence of small shifts about $4.6 \mathrm{MeV}=\Delta=9 m_{e}$ directly observed in the pion's mass splitting and $\tau$ lepton mass shift (boxed in Table 2). CODATA parameter $\delta=16 m_{e}$ can be considered as a result of the relation $1: 9=1:(3 \times(9 / 3=1 / 3+8 / 3))$ mentioned in [1-3] after comparison of the lepton ratio $L$ with number of fermions in the central field (Table 3). Integer relation $9=8+1$ could be connected with the new value of the principal quantum number in the fermion shell-like system (in accordance with V. Gribov suggestion that three colors are corresponding to three axes in the inner space):

$$
\begin{array}{rlrl}
\quad=(1 / 3) \mathrm{m}_{e}+(8 / 3) \mathrm{m}_{e} & & =(1 / 3) \mathrm{M}_{q}+(8 / 3) \mathrm{M}_{q} \\
9 \mathrm{~m}_{e}=(1 / 3) \mathrm{m}_{e}+(8 / 3) \mathrm{m}_{e} & 9 \mathrm{M}_{q} & =(1 / 3) \mathrm{M}_{q}+(8 / 3) \mathrm{M}_{q} \\
=(1 / 3) \mathrm{m}_{e}+(8 / 3) \mathrm{m}_{e} & & (1 / 3) \mathrm{M}_{q}+(8 / 3) \mathrm{M}_{q}
\end{array}
$$

Table 3. Comparison of ratios between masses $m_{m u} / M_{Z}, f_{\pi} /(2 / 3) m_{t}$ and $\Delta M_{H} / M_{H}$, QED parameter $\alpha / 2 \pi$ and numbers of fermions in the central field (central line, boxed in the bottom line is the hole configuration in $1 p$ shell).

\begin{tabular}{cccccc}
\hline N ferm. & $\mathrm{N}=1$ & 16 & $16 \cdot 13-1$ & $16 \cdot 16$ & $16 \cdot 18$ \\
\hline Part./par. & $m_{e} / M_{q}$ & $\delta / \delta^{\circ}$ & $\mathrm{m}_{\mu} / M_{Z}$ & $f_{\pi} /\left(M_{H}^{\prime}=2 m_{t} / 3=2 M^{L 3}\right.$ & $\Delta M_{\Delta} / M_{H}$ \\
\hline Ratio & $116 \cdot 10^{-5}$ & & $116 \cdot 10^{-5}$ & $116 \cdot 10^{-5}$ & $116 \cdot 10^{-5}$ \\
$\mathrm{Nr}$ & $(1 / 16)$ & $(1)$ & $12+1$ & 16 & 18 \\
\hline States & $1 s_{1 / 2}^{4}$ & $1 s_{1 / 2}^{4}, 1 p_{3 / 2}^{8}, 1 p_{1 / 2}$ & $1 s_{1 / 2}^{4}, 1 p_{3 / 2,1 / 2}^{8,4}$ & \\
\hline Comm. & & hole in $1 p$ shell & filled shells \\
\hline
\end{tabular}

Performed here and in [1-5] analysis of particle masses and nuclear data confirmed the presence of the so-called CODATA relation with the fine structure parameters $161 \mathrm{keV}=\delta m_{N} / 8$ and 170 $\mathrm{keV}=m_{e} / 3$ as well as the scaling factor $\alpha / 2 \pi$ equal to QED radiative correction. Possible origin of the baryon number and the color is due to involvement of $1 / 3$ part of the electron mass. Universal character of the electric charge and the spin should be theoretically combined with observed empirical CODATA-relations. These correlations found long ago [9] are confirmed now with analysis of new nuclear data [1]. The role of nuclear data in the study of tuning effect is very important.

\section{References}

[1] S.I. Sukhoruchkin, Proc. 19th Int. Conf. QCD-16, Montpellier; Nucl. Part. Phys. Proc. (in press).

[2] S.I. Sukhoruchkin, Nucl. Particle Physics Proc. 270-272 (2016) 211.

[3] S.I. Sukhoruchkin, Nucl. Part. Phys. Proc. 258-259 (2015) 268.

[4] S.I. Sukhoruchkin, Nucl. Phys. B (Proc. Suppl.) 234 (2013) 241.

[5] S.I. Sukhoruchkin, PoS (EPS-HEP2015) 527.

[6] F. Wilczek, Nature 520 (2015) 303.

[7] J. Gasser and H. Leutwyler, Phys. Rep. 87 (1982) 77.

[8] S.I. Sukhoruchkin, Prog. and Theses, 40-th Meet. Nucl. Spectroscopy, Leningrad, 1990. Nauka, p.147.

[9] S.I. Sukhoruchkin, Symmetry Methods in Physics, JINR E2-94-347, vol. 2, p. 528 (1994). 